\title{
Prevalencia de la Arteria Estriada Medial Distal en una Muestra de Poblaciones Colombiana y Peruana
}

\author{
Prevalence of Distal Medial Striatal Artery in a Sample of Colombian and Peruvian Populations
}

\author{
Karen D. Sanabria-Pinilla1; Yobany Quijano-Blanco' ${ }^{1}$ \& Vladimir A. Huayta-Alarcón²
}

SANABRIA-PINILLA, K. D.; QUIJANO-BLANCO, Y. \& HUAYTA-ALARCÓN, V. A. Prevalencia de la arteria estriada medial distal en una muestra de poblaciones colombiana y peruana. Int. J. Morphol., 37(3):997-1002, 2019.

RESUMEN: La arteria estriada medial distal hace parte de la circulación encefálica, nace de la arteria cerebral anterior generalmente a nivel de la arteria comunicante anterior, aunque según distintos estudios su origen varía, siendo así difícil de determinar con exactitud. Su importancia clínica radica en la prevalencia de aneurismas encontrados en esta arteria, que posteriormente podrían causar complicaciones debido a la región que irrigan, siendo estas las secuelas somático-vitales y neuropsicológicas, además de su inadecuado abordaje quirúrgico sin prever las alteraciones que puedan ser ocasionados; a causa de lo anterior es de vital importancia que los profesionales de la salud tengan previo conocimiento de la anatomía y la prevalencia de esta arteria en la población. Se realizó un estudio observacional de tipo descriptivo en donde se analizó la arteria estriada medial distal en 70 encéfalos, piezas de los anfiteatros de Medicina de la Universidad de Ciencias Aplicadas y Ambientales U.D.C.A. (Bogotá), la Universidad Científica del Sur UCSUR (Lima); fijados en formol al $10 \%$, se realizaron mediciones morfométricas mediante calibrador digital y se tomó el registro fotográfico con una cámara Canon. Posteriormente se ejecutó el análisis estadístico mediante el programa IBM SPSS Stadistics 24. Se encontró una prevalencia del 88,6 \% de al menos una arteria estriada medial distal en población colombiana y un 97,1 \% de la población peruana. Se identificó un diámetro externo promedio de $0,64 \mathrm{~mm}$ en población colombiana y de $0,68 \mathrm{~mm}$ en población peruana. Se observó una longitud promedio de 2,5 cm en ambas poblaciones. Se evidenció el mayor lugar de origen en la porción A2 de la ACA con un 37,1 \% de población colombiana y un $51,4 \%$ de población peruana. Se debe conocer adecuadamente la anatomía y las correspondientes variaciones anatómicas de esta arteria para así poder realizar un adecuado abordaje neurológico y neuroquirúrgico.

PALABRAS CLAVE: Arterias cerebrales; Anatomía regional; Neuroanatomía; Neurocirugía.

\section{INTRODUCCIÓN}

La arteria estriada medial distal, primero denominada la arteria recurrente de Heubner, fue descrita por primera vez en 1872 por el médico pediatra alemán Johann Otto Leonhard Heubner, quien la refirió como una arteria que se origina de la arteria cerebral anterior y la cual irriga la cabeza del núcleo caudado (Gasca-González et al., 2011). Esta arteria se describió por primera vez en letra impresa como "Arteria de Heubner" por Aitken, un artista en el Hospital general de Massachusetts en 1909 quien durante 5 años realizó disecciones anatómicas detalladas de la circulación cerebral de 45 cerebros humanos, posteriormente concluyó que la descripción de Otto Heubner era más precisa que la que había realizado Henri Duret en el Hospital de Salpetriere de Paris. Posteriormente a la descripción por Aitken y otros autores fue identificada como "Recurrente" por Joseph Schellshear en el Hospital St. Bartholomew de Londres en 1920, el cual realizó estas disecciones para establecer el significado funcional de las arterias basales y tratar de resolver las discrepancias resultantes de investigaciones pasadas (Haroun et al., 2000).

Otros autores han llamado a este vaso, arteria central larga, arteria telencefálica, arteria estriada rostral y más recientemente arteria estriada medial distal (Maga et al, 2013a).

La arteria estriada medial distal hace parte de la circulación encefálica, nace de la arteria cerebral anterior (A.C.A.) generalmente a nivel de la arteria comunicante

\footnotetext{
${ }^{1}$ Departamento de Anatomía Human, Facultad de Ciencias de la Salud,Programa de Medicina, Universidad de Ciencias Aplicadas y Ambientales. Bogotá, Colombia.

${ }^{2}$ Departamento de Morfofisiología. Facultad de Ciencias de la Salud. Escuela de medicina. Universidad Científica del Sur. Lima, Perú.
} 
anterior (ACoA) (Latarjet et al., 2004) se ha descrito que esta arteria se dirige hacia la sustancia perforada anterior donde termina sus segmento extracerebral y posteriormente se introduce en el parénquima (Gasca-González et al.).

La arteria estriada medial distal, suele ser la arteria de mayor calibre de las lenticuloestriadas medianas perforantes, y suele ramificarse tanto desde la porción A1, la porción A2, o en la unión entre la Arteria cerebral media y la arteria comunicante anterior (Loukas et al., 2006; Snell, 2010) Hay que tener en cuenta la posibilidad de anastomosis con la arteria cerebral media, ya que se ha descrito esta posterior a sus manifestaciones clínicas (Mavridis \& Anagnostopoulou, 2010).

Esta arteria realiza un trayecto recurrente y antes de profundizarse da ramas colaterales que irrigan a la corteza frontal y al tracto olfatorio (Latarjet et al.). Cuando llega a la sustancia perforada anterior penetra en ella por delante de las ramas profundas de la arteria cerebral media, termina en la cabeza del núcleo caudado y el brazo anterior de la cápsula interna, irrigando así a regiones del putamen y el globo pálido cuando se encuentra en su trayecto intraparenquimatoso (Toyoda, 2012). A su vez también proporciona irrigación a las porciones posteriores del giro recto y la corteza orbitofrontal.

A pesar de que su anatomía se encuentra descrita de manera general, se ha encontrado reportes de caso y descripciones anatómicas las cuales indican que la frecuencia de esta, su ubicación y su irrigación es muy variada a nivel mundial; se cuenta con estudios realizados en continente europeo y en el continente americano las bases de datos son mínimas solo con estudios realizados en México y Brasil, y no se tienen estudios en nuestra población Colombiana ni estudios en Perú, lo que conlleva a desconocer las alteraciones, la frecuencia y la ubicación de esta arteria, siendo de gran importancia especialmente en el campo de la neurología y la neurocirugía conocer la prevalencia y sus alteraciones para así realizar un correcto abordaje de las patologías neurológicas y evitar complicaciones en las correcciones neuroquirúrgicas.

La importancia clínica de la arteria estriada medial distal radica en el desconocimiento de la prevalencia y las complicaciones posteriores a clipajes de aneurismas de la arteria comunicante anterior lo cual puede ocasionar durante las intervenciones quirúrgicas complicaciones como obstrucción o daños a la irrigación, ocasionado por la vinculación con el tronco de la arteria comunicante anterior o con el desconocimiento de variaciones de la arteria estriada medial distal a nivel de la arteria comunicante anterior (Maga et al., 2013b; El Falougy et al., 2013). Además de producir- se infartos del estrato mediobasal asociado a trombosis de la Arteria estriada medial distal, descritos en 6 casos de 400 autopsias, y solo en uno de estos casos se estableció la patogenia (Gomes et al., 1984).

Adicionalmente se encuentran malformaciones arteriovenosas y en especial los aneurismas encontrados en esta arteria, muchos de ellos asociados a neurofibromatosis de tipo 1 (Gasca-González et al.; Tamez-Montes et al., 2012) y a osteogénesis imperfecta (Mansfield \& Rahme, 2015), que posteriormente podrían causar complicaciones debido a la región que irrigan, siendo estas las secuelas somáticovitales y neuropsicológicas así como afasias, trastornos de conducta, hemiparesias dominantemente faciobraquial y hasta la muerte (Cohen et al., 2012; Yilmaz et al., 2016).

Por lo anterior es de motivación conocer la anatomía extra e intracerebral además de las variaciones de la arteria estriada medial distal en nuestra población y compararla con otra población latinoamericana con el fin de tener en cuenta prevención, diagnóstico y tratamiento oportuno de las patologías ocasionadas a alteraciones en esta arteria.

\section{MATERIAL Y MÉTODO}

Se realizó un estudio descriptivo de corte transversal con 70 encéfalos que se encontraban en la Universidad de Ciencias Aplicadas y Ambientales U. D. C. A. (Bogotá, Colombia) y la Universidad Científica del Sur UCSUR (Lima, Perú), se realizó un muestreo a conveniencia teniendo en cuenta como criterios de inclusión que el material estuviera fijado en formaldehído al $10 \%$ y fueran encéfalos completos, y como criterios de exclusión el material que presentara daños estructurales observados macroscópicamente y/o daños en la circulación encefálica.

Se realizó la recolección de datos, teniendo en cuenta que los 70 encéfalos se encontraran debidamente marcados y rotulados (Figs. 1 y 2) que se encontraban fijados con formaldehido al $10 \%$, posteriormente se realiza el abordaje mediante disección con el fin de determinar la incidencia, la presencia de ramas, el lugar de origen y la observación de variaciones en estas; posteriormente mediante el uso de un calibrador digital se procedió a realizar mediciones de estas arterias (longitud extracerebral y diámetro externo de la arteria), realizando así el registro fotográfico.

Adicionalmente para dar calidad del dato se realizaron cinco procesos, en los cuales se encuentra aseguramiento de la calidad, se entrenó al equipo para la recolección de datos con este instrumento. Para el control de calidad se 
observó el procedimiento de medición por cada miembro del equipo, en donde se evalúo el tiempo empleado en estas, además de la comparación con los otros observadores.

En el manejo de datos se registraron inicialmente en el sitio de observación en la matriz de variables propuesta, luego en este mismo sitio se trasladaron los datos a Software IBM SPSS Stadistics 24 conformando así la base de datos.

Se realizó análisis estadístico mediante el programa IBM SPSS Stadistics 24 teniendo en cuenta la matriz de variables propuesta (Variables prevalencia, longitud extracerebral, diámetro externo, $\mathrm{N}^{\circ}$ de ramas y presencia de variaciones); a partir de este se realizó el análisis clínico de cada población, análisis comparativo y la elaboración de conclusiones.

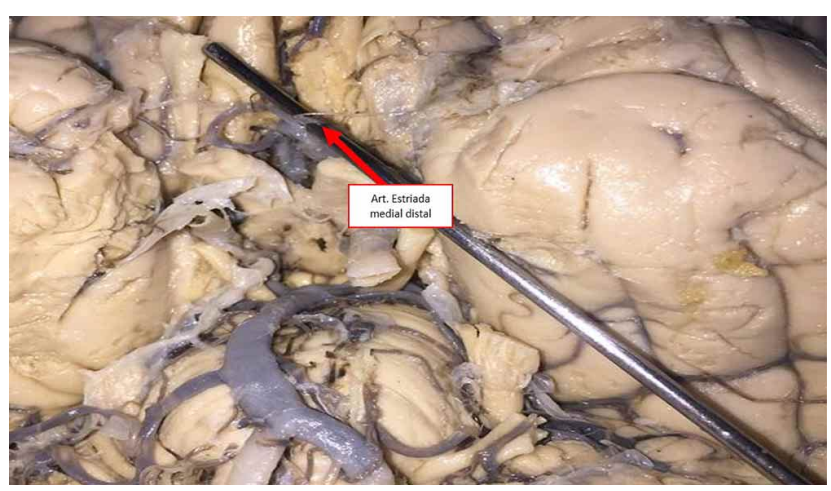

Fig. 1. Registro de la Arteria estriada medial distal, rama única. Imagen tomada en Anfiteatro de Medicina Humana UCSUR.

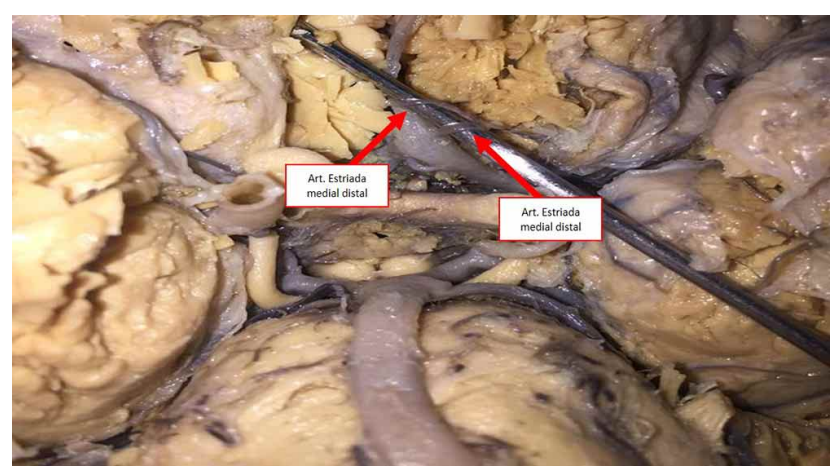

Fig. 2. Registro de la Arteria estriada medial distal, rama doble. Imagen tomada en Anfiteatro de Medicina Humana UCSUR.

\section{RESULTADOS Y DISCUSIÓN}

Se encontró al menos una arteria estriada medial distal en alguno de los hemisferios de la población colombiana en un $88,6 \%$ (31 encéfalos) y en un $97,1 \%$ de la población peruana (34 encéfalos), siendo prevalente en las dos pobla- ciones su presencia en ambos hemisferios con un 51,4\% y un $77,1 \%$ respectivamente, seguido de la presencia de manera unilateral en hemisferio izquierdo en Colombia (22,9 $\%)$ y unilateral en el hemisferio derecho en Perú $(11,4 \%)$ y con una ausencia del $11,4 \%$ en Colombia y $2,9 \%$ en población peruana. El número de ramas encontrado en el hemisferio derecho peruano fue de mayor prevalencia una rama $(68,6 \%)$ seguido de dos ramas con un $22,9 \%$ y ausencia de ramas en un 8,6\%; en el hemisferio izquierdo peruano fue prevalente la presencia de una rama con un $51.4 \%$, con dos ramas se encontró un $34,3 \%$ y ausencia de ramas con un $14,3 \%$. En la población colombiana se observó en el hemisferio izquierdo la presencia de una rama con un 54,3\%, seguido en frecuencia la ausencia de ramas con un $25,7 \%$, la presencia de dos ramas con un $17,1 \%$ y se encontró un hallazgo particular de la observación de una tercera rama en uno de los hemisferios evaluados (2,9\%) (Figs. 3). En el hemisferio derecho colombiano se presentó una mayor prevalencia con una rama en un $48,6 \%$, seguido por la ausencia de ramas con un $34,3 \%$ y con dos ramas en un $17,1 \%$.

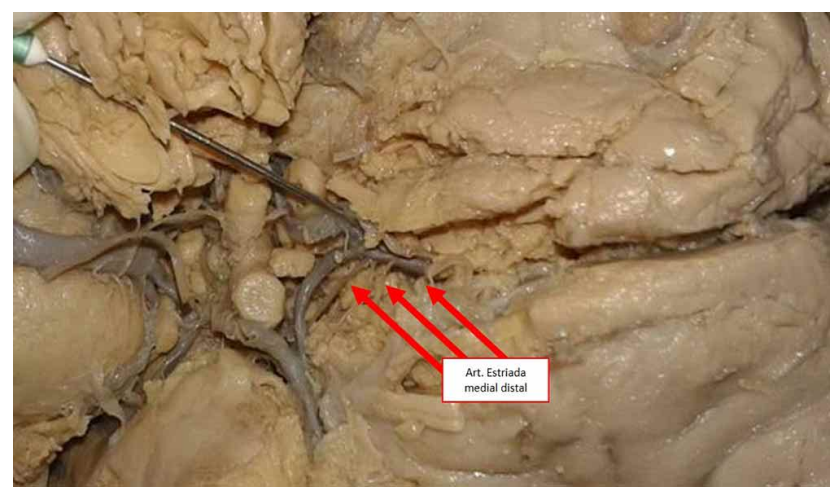

Fig. 3. Registro de la Arteria estriada medial distal, rama triple. Imagen tomada en Anfiteatro de Medicina Humana U.D.C.A.

En cuanto a la medición del diámetro externo de la arteria estriada medial distal se estimó que en población peruana en el intervalo de 5,8 a $6,9 \mathrm{~mm}$ se tuvo la mayor prevalencia con un $20 \%$, y se registró la menor prevalencia en el intervalo de 7,0 a 8,1 con un $2,9 \%$. El diámetro mínimo observado fue de $1,4 \mathrm{~mm}$ y el diámetro externo máximo en $18,4 \mathrm{~mm}$ con un promedio de $6,8 \mathrm{~mm}$. En cuanto a la población colombiana el intervalo con mayor prevalencia fue de 4,5 a $5,3 \mathrm{~mm}$ con un $17,1 \%$, se observó un diámetro mínimo de 3,4 mm y un máximo de 9,8 $\mathrm{mm}$ con un promedio de $6,4 \mathrm{~mm}$.

En la medición de la longitud extracerebral de la arteria estriada medial la población peruana tuvo con un $25,7 \%$ la mayor prevalencia que se encontraba en el intervalo de 2,35 a $2,72 \mathrm{~cm}$ y el menor intervalo entre 3,84 
SANABRIA-PINILLA, K. D.; QUIJANO-BLANCO, Y. \& HUAYTA-ALARCóN, V. A. Prevalencia de la arteria estriada medial distal en una muestra de poblaciones colombiana y peruana. Int. J. Morphol., 37(3):997-1002, 2019.

Tabla I. Resultado de investigaciones relacionados con la arteria estriada medial distal

\begin{tabular}{|c|c|c|c|c|c|c|c|}
\hline \multirow[t]{2}{*}{ VARIABLE } & $\begin{array}{l}\text { Loukas et al. } \\
\text { Antillas } \\
\text { Holandesas }\end{array}$ & $\begin{array}{l}\text { Maga et al. } \\
\text { Polonia }\end{array}$ & $\begin{array}{l}\text { El Falougy et al. } \\
\text { Eslovaquia }\end{array}$ & $\begin{array}{l}\text { Gasca- } \\
\text { González et al., }\end{array}$ & $\begin{array}{l}\text { Castillejos, } \\
\text { Grapiglia y Cols. }\end{array}$ & $\begin{array}{l}\text { Sanabria et al.. } \\
\text { Colombia }\end{array}$ & $\begin{array}{l}\text { Sanabria et al. } \\
\text { Perú }\end{array}$ \\
\hline & & & & México & Brasil & (trabajo actual) & (trabajo actual) \\
\hline \multicolumn{8}{|l|}{ PRESENCIA } \\
\hline \multirow{2}{*}{$\begin{array}{l}\text { Presencia de } \\
\text { único }\end{array}$} & $77 \%$ & $29.7 \%$ & $88,5 \%$ & $54 \%$ & $96,6 \%$ & HD $48,6 \%$ & HD $65,7 \%$ \\
\hline & & & & & & HI 54,3\% & HI $51,4 \%$ \\
\hline \multirow[t]{2}{*}{ Presencia vaso doble } & $17 \%$ & $43,6 \%$ & $3,55 \%$ & $39 \%$ & $1,6 \%$ & HD 17,1\% & HD 22,9 \% \\
\hline & & & & & & HI $17,1 \%$ & HI $34,3 \%$ \\
\hline Presencia vaso triple & - & $24,6 \%$ & $2,7 \%$ & - & $1,6 \%$ & HI $2,9 \%$ & - \\
\hline \multirow{3}{*}{$\begin{array}{l}\text { Presencia vaso con } \\
\text { más ramas } \\
\text { Ausencia }\end{array}$} & - & $2,2 \%$ & - & - & - & - & - \\
\hline & $6 \%$ & - & $5,19 \%$ & $7 \%$ & $1,6 \%$ & HD $34,3 \%$ & HD $11,4 \%$ \\
\hline & & & & & & HI $25,7 \%$ & HI 14,3\% \\
\hline \multicolumn{8}{|l|}{ ORIGEN } \\
\hline Unión ACA-ACoA & $62,3 \%$ & $40 \%$ & $43,4 \%$ & $41,4 \%$ & $23,4 \%$ & $14,3 \%$ & $11 \%$ \\
\hline A1 & $11 \%$ & $26,2 \%$ & $3,55 \%$ & $6,35 \%$ & $16,6 \%$ & $20 \%$ & $11 \%$ \\
\hline $\mathrm{A} 2$ & $18 \%$ & $33,8 \%$ & $47,81 \%$ & $45,1 \%$ & $60 \%$ & $37,1 \%$ & $51,4 \%$ \\
\hline Art, Frontopolar & - & - & - & $14,3 \%$ & - & - & - \\
\hline Doble origen & - & - & - & - & - & $17,1 \%$ & $22,9 \%$ \\
\hline \multicolumn{8}{|l|}{ A2/ACA-ACoA } \\
\hline Ausencia de origen & - & - & - & - & - & $11,5 \%$ & $3,7 \%$ \\
\hline \multicolumn{8}{|l|}{ Trayecto } \\
\hline Superior & - & $61 \%$ & - & - & $74,2 \%$ & - & - \\
\hline Anterior & - & $32 \%$ & - & - & $22,6 \%$ & - & - \\
\hline Inferior & $4 \%$ & - & - & $1,6 \%$ & & - & - \\
\hline Posterior & - & $3 \%$ & - & - & $1,6 \%$ & - & - \\
\hline DIÁMETRO & $8 \mathrm{~mm}$ & $2 \mathrm{~mm}$ & $6 \mathrm{~mm}$ & - & $3 \mathrm{~mm}$ & $6,4 \mathrm{~mm}$ & $6,8 \mathrm{~mm}$ \\
\hline LONGITUD & $2,3-2,5 \mathrm{~cm}$ & $2,52 \mathrm{~cm}$ & - & $2,52 \mathrm{~cm}$ & $2,5 \mathrm{~cm}$ & $2,5 \mathrm{~cm}$ & $2,5 \mathrm{~cm}$ \\
\hline \multicolumn{8}{|l|}{ TRAYECTORIA } \\
\hline $\mathrm{L}$ & - & - & - & $32,5 \%$ & - & - & - \\
\hline L Invertida & - & - & - & $14,5 \%$ & - & - & - \\
\hline Oblicua & - & - & - & $32,3 \%$ & - & - & - \\
\hline Sinuosa & - & - & - & $20,75 \%$ & - & - & - \\
\hline
\end{tabular}

y más con un $2,9 \%$ con una longitud mínima de $1,08 \mathrm{~cm}$ localizada en el hemisferio derecho, una longitud máxima de $3,84 \mathrm{~cm}$ observada en el hemisferio izquierdo y un promedio de $2,50 \mathrm{~cm}$, en la discriminación por hemisferios, en el hemisferio izquierdo se obtuvo un $25,7 \%$ en el intervalo de 2,35 a $2,72 \mathrm{~cm}$ y el menor intervalo estuvo entre 3,84 y más con un $2,9 \%$ con una longitud mínima de $1,39 \mathrm{~cm}$ y máxima de 3,84 $\mathrm{cm}$, además de un promedio de $2,48 \mathrm{~cm}$, en el hemisferio derecho se tuvo una prevalencia de $34,3 \%$ presente en el intervalo de 2,46 a $2,79 \mathrm{~cm}$ y la menor prevalencia se encontró en los intervalos de 0,78 a $1,11 \mathrm{~cm}$ y de 1,12 a $1,44 \mathrm{~cm}$ con un 2,9 $\%$ en estos. Se identificó una longitud mínima de 1,08 cm una longitud máxima de $3,46 \mathrm{~cm}$ con un promedio de $2,51 \mathrm{~cm}$.
En la población colombiana se encontró en mayor prevalencia con un porcentaje de $37,1 \%$ en el intervalo de 2,09 a $2,41 \mathrm{~cm}$ y los intervalos con menor prevalencia con un $2,9 \%$ fueron entre 1,76 y $2,08 \mathrm{~cm}$ y entre $3,08 \mathrm{~cm}$ y mayores medidas. Se observó una longitud mínima de $1,39 \mathrm{~cm}$ en el hemisferio derecho y una longitud máxima de $3,42 \mathrm{~cm}$ en el mismo hemisferio, y un promedio de $2,5 \mathrm{~cm}$. Realizando la discriminación por hemisferios, en el hemisferio derecho con un 34,3 $\%$ en el intervalo menor de $1,10 \mathrm{~cm}$ y con una menor prevalencia con un 2,9\% en los intervalos de 1,11 a 1,43 cm; de 1,44 a $1,76 \mathrm{~cm}$; de 1,77 a 2,09 $\mathrm{cm}$ y de medidas mayores a $3,10 \mathrm{~cm}$. En este hemisferio se tiene una longitud mínima de $1,39 \mathrm{~cm}$ y una máxima de $3,42 \mathrm{~cm}$ y un promedio de $2,46 \mathrm{~cm}$. 
En el hemisferio izquierdo se encuentra en una mayor prevalencia en el intervalo entre 2,09 y $2,41 \mathrm{~cm}$ con un $37,1 \%$ y con la menor prevalencia en los intervalos de 1,76 a 2,08 cm y de $3,08 \mathrm{~cm}$ y medidas mayores con un $2,9 \%$, con una longitud máxima de $3,40 \mathrm{~cm}$ y una mínima de $2,01 \mathrm{~cm}$ y un promedio de $2,01 \mathrm{~cm}$.

De acuerdo a la observación del sitio de origen de la AEMD en la población peruana se registra la mayor prevalencia en el segmento A2 con un 51,4\% (18 encéfalos), seguido del origen en ramas dobles (Una rama en A2 y una rama en ACA-ACoA) con un 22,9\% que corresponde a 8 encéfalos , posteriormente el origen en la unión ACA-ACoA y en el segmento A1 con un 11,4\% (4 encéfalos); en la población colombiana se observó en él una prevalencia mayor con un $37,1 \%$ en el segmento A2 (13 encéfalos), seguido de la prevalencia en el segmento A1 con un porcentaje de $20 \%$ (7 encéfalos), le sigue el origen en ramas dobles (Una rama en A2 y una rama en ACA-ACoA) con un 17,1 \% (6 encéfalos) y por último el origen en la unión ACA-ACoA con un 14,3 \% (5 encéfalos), se realiza una prueba de chi cuadrado dando como resultado un valor de 0,431 , por lo cual se acepta la hipótesis nula, indicando que el país no modifica el origen de la arteria estriada medial distal.

Se evidencia que, en comparación con las investigaciones realizadas anteriormente (Tabla I), nosotros realizamos la discriminación por hemisferios para la realización del estudio, en cuanto a la presencia de único vaso se tienen valores relacionados (tanto en población peruana como en población colombiana) con la investigación de Gasca-González et al. en México, en la presencia de vaso doble en población colombiana se observa hallazgos similares a los obtenidos en la investigación realizada por Loukas et al. en las Antillas Holandesas, mientras que los valores registrados para población peruana son similares al estudio realizado en México. Se evidencia que la presencia de vaso triple se registró en nuestra población colombiana tiene relación con la registrada en Brasil y en Eslovaquia; en cuanto a la ausencia de vasos se observó una mayor prevalencia en población colombiana a comparación de las demás investigaciones, sin embargo, se observa en la investigación realizada en México el valor en ascenso en comparación con las demás investigaciones lo que puede estar en relación con la población de estudio.

En relación con el origen de la arteria estriada medial distal se observa hallazgos en relación con el nacimiento en la unión ACA-ACoA menores en comparación con los demás estudios, a pesar de esto, se evidencia valor cercano en la población brasilera estudiada. En cuanto a la relación de origen en la región A1 de la arteria cerebral anterior se observan valores relacionados en todas las investigaciones incluyendo la nuestra, en relación con el nacimiento en la región A2 de la arteria cerebral anterior también se evidencian valores similares en comparación de las investigaciones anteriores a excepción de la realizada en las Antillas Holandesas donde se obtuvo una frecuencia menor. En nuestra investigación se tuvo en cuenta el nacimiento en dos regiones cuando se tenían ramas múltiples de la Arteria estriada medial distal, en donde las únicas ubicaciones registradas era el nacimiento de una arteria en la región A2 de la arteria cerebral anterior y de la otra en la unión entre la arteria cerebral anterior y la arteria comunicante anterior teniendo como único dato en investigaciones a nivel internacional que se da en el $17,1 \%$ de la población colombiana y en un $22,9 \%$ de población peruana. Además, en nuestro estudio no se realizó la exclusión para estos datos de hemisferios con ausencia de arteria, por lo cual se contó con ausencia de origen en un 11,5\% de población colombiana y un $3,7 \%$ de población peruana.

Se observó el diámetro promedio de las poblaciones en donde se tienen valores similares a investigaciones realizadas en las Antillas Holandesas y en Eslovaquia, y con ligeras diferencias en investigaciones realizadas en Brasil y Polonia.

En cuanto a la longitud observada no hay diferencias mayores en ninguna de las investigaciones observadas incluyendo la nuestra.

Se recomienda para la próxima realización de estudios contar con una técnica de inyección arterial para lograr más adecuadamente la observación, se requieren realización de disección arterial en próximas investigaciones ya que al realizar observación no es posible visualizar la forma de recorrido extracerebral, además se sugieren estudios de neuroimagen en donde se observen los recorridos a nivel intracerebral. Se sugiere la realización de comparaciones por sexo ya que en este estudio no fue posible determinarlo, ni se contaba con él y por lo tanto se tuvo ausencia de este dato en los resultados.

CONSIDERACIONES ÉTICAS. Teniendo en cuenta la resolución 8430 de 1993 en su artículo 16 se establece que esta investigación es de riesgo mínimo, por lo cual exime a los investigadores de la realización de consentimiento informado (Ministerio de Salud, 1993). Se examinó la declaración de Helsinki para la investigación médica donde se garantiza un trato digno y respetuoso de las piezas obtenidas, además de guardar confidencialidad de los datos de la persona fallecida (Asociación Médica Mundial, 2013). Compendio de Normativa Ética para uso por los Comités de Ética en Investigación del 2011 en Perú, donde se declara seguir los lineamientos dispuestos en la investigación para ser ética y científicamente avalada (Ministerio de Salud del Perú, 2011). 


\section{AGRADECIMIENTOS}

Al Departamento de Anatomía Humana de la Universidad de Ciencias Aplicadas y Ambientales (Bogotá, Colombia), al departamento de morfofisiología humana de la Universidad Científica del Sur (Lima, Perú), por permitir la realización de esta investigación.

SANABRIA-PINILLA, K. D.; QUIJANO-BLANCO, Y.\& HUAYTA-ALARCÓN, V. A. Prevalence of distal medial striatal artery in a sample of Colombian and Peruvian populations. Int. J. Morphol., 37(3):997-1002, 2019.

SUMMARY: The distal medial striate artery is part of the brain circulation, born from the anterior cerebral artery generally at the level of the anterior communicating artery, although according to different studies its origin varies, being thus difficult to determine with accuracy. Its clinical importance lies in the prevalence of aneurysms found in this artery, which could later cause complications due to the region they irrigate, these being the somatic-vital and neuropsychological sequelae, in addition to its inadequate surgical approach without foreseeing the alterations that may be caused; Because of the above it is of vital importance that health professionals have prior knowledge of the anatomy and prevalence of this artery in the population. An observational descriptive study was carried out in which the distal medial striated artery in 70 brain cells, pieces from the Medicine amphitheatres of the Universidad de Ciencias Aplicadas y Ambientales U.D.C.A. (Bogotá), the Universidad Científica del Sur UCSUR (Lima); fixed in $10 \%$ formalin, morphometric measurements were made by automatic calibrator and the photographic record was taken with a Canon camera. Subsequently, the statistical analysis was executed through the IBM SPSS Statistics program 24. A prevalence of $88.6 \%$ of at least one distal medial striate artery was found in the Colombian population and $97.1 \%$ of the Peruvian population. An average external diameter of $0.64 \mathrm{~mm}$ was identified in the Colombian population and $0.68 \mathrm{~mm}$ in the Peruvian population. An average length of $2.5 \mathrm{~cm}$ was observed in both populations. The largest place of origin was evidenced in the A2 portion of the ACA with $37.1 \%$ of the Colombian population and $51.4 \%$ of the Peruvian population. The anatomy and the corresponding anatomical variations of this artery must be adequately known to be able to perform an adequate neurological and neurosurgical approach.

KEY WORDS: Cerebral arteries; Regional anatomy; Neuroanatomy; Neurosurgery.

\section{REFERENCIAS BIBLIOGRÁFICAS}

Asociación Médica Mundial. Declaración de Helsinki. Principios Éticos para Investigaciones Médicas en Seres Humanos. Ferney-Voltaire, Asociación Médica Mundial, 2013. Available from: http://www.isciii.es/ISCIII/ es/contenidos/fd-investigacion/fd-evaluacion/fd-evaluacion-eticainvestigacion/Declaracion-Helsinki-2013-Esp.pdf

Cohen, J. E.; Rabinstein, A.; Gomori, J. M. \& Leker, R. R. Capsular warning syndrome and crescendo lacunar strokes after atherosclerotic stenosis of the recurrent artery of Heubner. J. Clin. Neurosci., 19(12):1730-3, 2012.

El Falougy, H.; Selmeciova, P.; Kubikova, E. \& Haviarová, Z. The variable origin of the recurrent artery of Heubner: an anatomical and morphometric study. Biomed. Res. Int., 2013:873434, 2013.

Gasca-González, O. O.; Delgado-Reyes, L. \& Pérez-Cruz, J. C. Anatomía microquirúrgica del segmento extracerebral de la arteria recurrente de Heubner en población mexicana. Cir. Cir., 79(3):219-24, 2011.

Gomes, F.; Dujovny, M.; Umansky, F.; Ausman, J. I.; Diaz, F. G.; Ray, W. J. \& Mirchandani, H. G. Microsurgical anatomy of the recurrent artery of Heubner. J. Neurosurg., 60(1):130-9, 1984.

Haroun, R. I.; Rigamonti, D. \& Tamargo, R. J. Recurrent artery of Heubner: Otto Heubner's description of the artery and his influence on pediatrics in Germany. J. Neurosurg., 93(6):1084-8, 2000.

Latarjet, M.; Ruíz Liard, A. \& Pró, E. Anatomía Humana. $4^{\text {a }}$ ed. Buenos Aires, Médica Panamericana, 2004.

Loukas, M.; Louis, R. G. Jr. \& Childs, R. S. Anatomical examination of the recurrent artery of Heubner. Clin. Anat., 19(1):25-31, 2006.

Maga, P.; Tomaszewski, K. A.; Pasternak, A.; Zawilinski, J.; Tomaszewska, R.; Gregorczyk-Maga, I. \& Skrzat, J. Extra- and intracerebral course of the recurrent artery of Heubner. Folia Morphol. (Warsz), 72(2):94-9, 2013a.

Maga, P.; Tomaszewski, K. A.; Skrzat, J.; Tomaszewska, I. M.; Iskra, T.; Pasternak, A. \& Walocha, J. A. Microanatomical study of the recurrent artery of Heubner. Ann. Anat., 195(4):342-50, 2013b.

Mansfield, K. \& Rahme, R. Dissecting aneurysm of the recurrent artery of Heubner in a patient with osteogenesis imperfecta. Can. J. Neurol. Sci., 42(6):461-5, 2015.

Mavridis, I. \& Anagnostopoulou, S. Comment on the brain areas whose blood supply is provided by the recurrent artery of Heubner. Surg. Radiol. Anat., 32(1):91, 2010.

Ministerio de Salud. Instituto Nacional de Salud. Compendio de Normativa Ética para uso por los Comités de Ética en Investigación. Lima, Ministerio de Salud del Perú, 2011.

Ministerio de Salud. Resolución Número 8430 de 1993. Resolución número 8430 de 1993. Bogotá, Ministerio de Salud, República de Colombia, 1993. Available from: https://www.minsalud.gov.co/sites/rid/Lists/ BibliotecaDigital/RIDE/DE/DIJ/RESOLUCION-8430-DE-1993.PDF

Snell, R. Neuroanatomía Clínica. $7^{\text {th }}$ ed. Buenos Aires, Médica Panamericana, 2010.

Tamez-Montes, D.; Ramírez-Barrios, L. R.; Garza-Mercado, R.; MartínezPonce de León, Á. R.; Villarreal-Reyna, G. \& Peña-Ramírez, D. Tratamiento microquirúrgico de los aneurismas intracraneales rotos. Arch. Neurocienc., 17(1):39-41, 2012.

Toyoda, K. Anterior cerebral artery and Heubner's artery territory infarction. Front. Neurol. Neurosci., 30:120-2, 2012.

Yilmaz, A.; Kizilay, Z.; Ozkul, A. \& Çirak, B. Pure motor stroke secondary to cerebral infarction of recurrent artery of Heubner after mild head trauma: a case report. Open Access Maced. J. Med. Sci., 4(1):139-41, 2016.

Dirección para correspondencia:

Daniela Sanabria

Departamento de Anatomía Humana

Facultad de ciencias de la salud

Universidad de Ciencias Aplicadas y Ambientales

calle 222 No. 54-37

Bogotá - COLOMBIA

Email: ksanabria@udca.edu.co

Recibido : 26-09-2018

Aceptado: 05-02-2019 